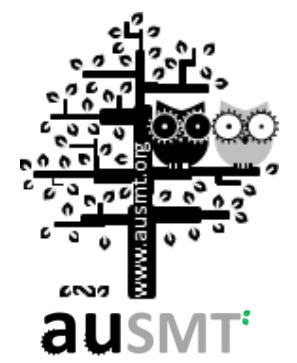

\title{
Digital Microfluidics with Bubble Manipulations by Dielectrophoresis
}

\section{Shih-Kang Fan* and Di-Yu Lin}

Department of Materials Science and Engineering, National Chiao Tung University, Taiwan

(Received 15 November 2011; Accepted 16 January 2012; Published on line 1 March 2012)

*Corresponding author: skfan@mail.nctu.edu.tw

DOI: 10.5875/ausmt.v2i1.120

\begin{abstract}
This paper presents basic bubble manipulations, including transporting, splitting, and merging, by dielectrophoresis (DEP) in an oil environment. In our presented method, bubbles are placed between parallel plates in an oil medium of a low vapor pressure, which eliminates the possibility of changing the gaseous composition of the bubble caused by evaporation of the medium. DEP has been previously investigated to actuate dielectric droplets and is adopted here to drive the oil environment as well as the immersed bubbles between parallel plates. In our experiment, air bubbles of $0.3 \mu$ were successfully transported in a 20 cSt silicone oil medium between a $75 \mu \mathrm{m}$-high parallel plate gap. In addition, $0.6 \mu \mathrm{l}$ air bubbles were successfully split into two $0.3 \mu \mathrm{l}$ air bubbles, and then merged again by DEP. These successful manipulations make digital gaseous lab-on-a-chip a reality.
\end{abstract}

Keywords: Bubble; Dielectrophoresis

\section{Introduction}

Since the first demonstration of a reconfigurable droplet driven by electrowetting [1] or electrowetting-on-dielectric (EWOD) [2], the use of digital microfluidics (DMF) $[3,4]$ has grown rapidly over the past decade, both through academic research on biochemical assays [5] and industrial prototyping for point of care tests [6]. For the ability to control individual droplets on a programmable electrode array, electrowetting provides reconfigurability, flexibility, and portability to perform on-chip chemical and biomedical protocols [7]. In addition to serving as microreactors, the diversity of available droplet materials has led to optical, electrical, and thermal applications commensurate with their appropriate physical properties. For example, droplet-based lenses [8, 9] and light regulators [10] have been fabricated by controlling the surface profile, position, or the dispersed particle arrangement of a droplet with the proper refractive index, while electrical or thermal properties can be used to make droplets that are able to conduct electrical current or heat [11].

In addition to driving droplets, manipulating bubbles in a liquid surrounding is another approach to working with DMF. EWOD was later adopted to transport, split, merge, and eliminate bubbles immersed in water between parallel plates [12]. Developing bubble manipulations has become fundamental to digital gaseous lab-on-a-chip. Although EWOD has been applied to bubble manipulations, actuating bubbles in an aqueous medium may change the compositions of the bubbles because of the high vapor pressure of water. The vapor pressure of water at $25^{\circ} \mathrm{C}$ is about 23.8 torr, which can be 9 orders of magnitude higher than that of oil (e.g., $10^{-8}$ torr for some vacuum pump oils). To realize a digital (bubble-based) gaseous lab-on-a-chip for gas analysis, an inert and stable bubble carrier is necessary. As such, using a low vapor pressure oil medium to manipulate bubbles is important. Since EWOD cannot change the surface wettability between solid surface and a dielectric liquid, driving a bubble in a non-conductive medium such 
as oil by EWOD is not achievable. In addition to moving bubbles in water, thermocapillary force has been used to actuate bubbles in oil by manipulating temperatures [13]. The light-induced optoelectronic force also drives bubbles in oil on a titanium oxidephthalocyanine (TiOPc)-based substrate [14]. However, to date bubble manipulations merely moved bubbles, splitting bubbles was not reported.

In our present research, we have achieved transporting, splitting, and merging non-conductive droplets, including decane, hexadecane, and silicone oil of different viscosities oil, by dielectrophoresis (DEP) [15, 16]. Here, we present the use of DEP force to drive silicone oil and demonstrate bubble manipulations, including transporting, merging and splitting, in a low vapor pressure oil environment.

(a)

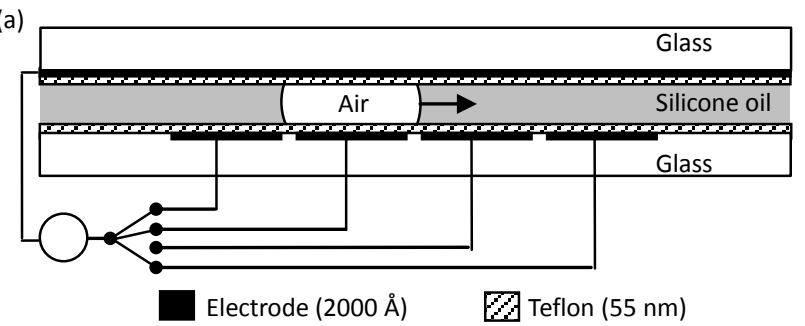

(b)



Figure1. Corss-sectional and top view of a DEP device to manipulate an air bubble in silicone oil between parallel plates. Electrodes are covered by Teflon to facilitate oil and bubble handling.

\section{Principle}

Drawing bulk dielectric liquids of higher relative permittivity (liquid) into a strong electric field region of lower relative permittivity (air) has been demonstrated

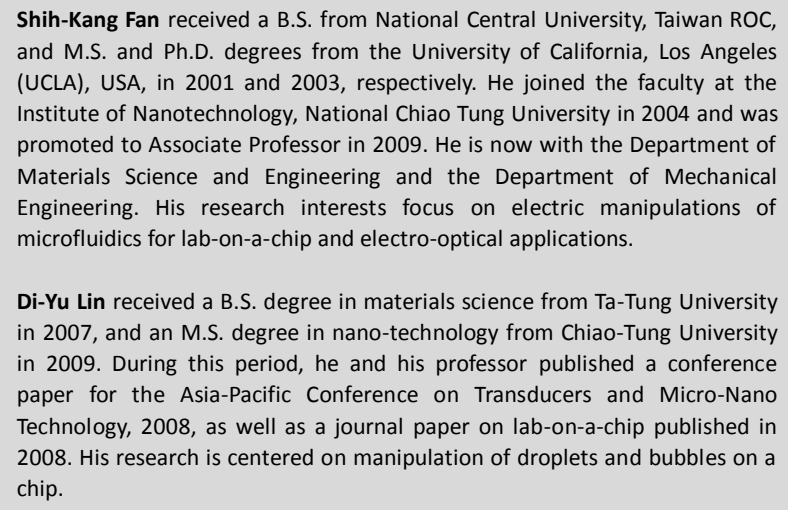

Di-Yu Lin received a B.S. degree in materials science from Ta-Tung University in 2007, and an M.S. degree in nano-technology from Chiao-Tung University in 2009. During this period, he and his professor published a conference paper for the Asia-Pacific Conference on Transducers and Micro-Nano Technology, 2008, as well as a journal paper on lab-on-a-chip published in 2008. His research is centered on manipulation of droplets and bubbles on a chip.

[17-20]. Over 100 years ago, raising dielectric liquid by applying a DC voltage between a pair of electrode plates dipped vertically into a dish of the dielectric liquid was achieved with a macroscopic apparatus [20]. The height-of-rise was experimentally and theoretically explained by force balance between gravity and the DEP force $\left(F_{D E P}\right)$ in the direction normal to the surface acting from the dielectric liquid to air. By applying $V$ between $W$-wide parallel electrode plates spaced $d$ apart, $F_{D E P}$ is:

$$
F_{D E P}=\frac{\varepsilon_{0}(\varepsilon-1) W}{2 d} V^{2}
$$

where $e_{0}\left(8.85 \times 10^{-12} \mathrm{~F} / \mathrm{m}\right)$ is the permittivity of a vacuum, and $e$ is the relative permittivity of the liquid. The DEP force has been successfully used to actuate dielectric droplets including silicone oil droplets $[15,16]$.

By actuating the silicone oil surrounded medium, DEP could push the bubbles away from the energized electrodes. As shown in Figure 1, an air bubble is immersed in silicone oil between Teflon-coated parallel glass plates. By applying a sufficient voltage between the top plate and bottom plate to actuate the bubble, silicone oil can be attracted to the energized electrodes and, in doing so, push the air bubble away from the energized electrodes. This means that the size of bubble would be affected by the neighboring electrode's size. Using Figure 1 as a guide, to push a bubble to the right side, the right electrode should be switched off, while other electrodes are turned on. However, since the bubble is pushed by DEP, the bubble would move slightly upward or downward as indicated by the arrows in Figure 1(b).

\section{Experiment}

To overcome the uncertainty of motion, an electrode is added that surrounds the bubble, as shown in Figure 2. This surrounding electrode is always energized to confine the bubble inside it. As shown in Figure 2(a), when applying voltage on the appropriate electrodes inside the confined area and under the bubble, the bubble is pushed to the right by DEP. Basically, the bubble is prone to move onto a non-energized electrode (labeled "Off" in Figure 2). Similarly, splitting a bubble can be achieved by turning off the two adjacent electrodes to the bubble and then turning on the electrode where the bubble is located, as shown in Figure 2(b). Merging bubbles, then, can be achieved by turning off the electrode where the bubble merging will take place and turning on the necessary electrodes to push the bubbles, as shown in Figure 2(c). 


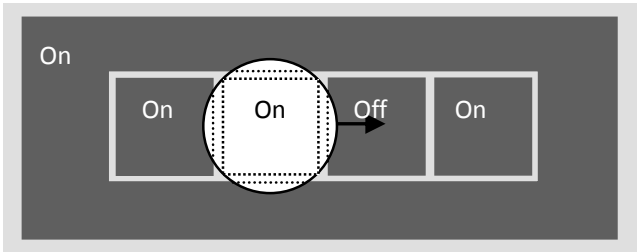

(a) Transporting

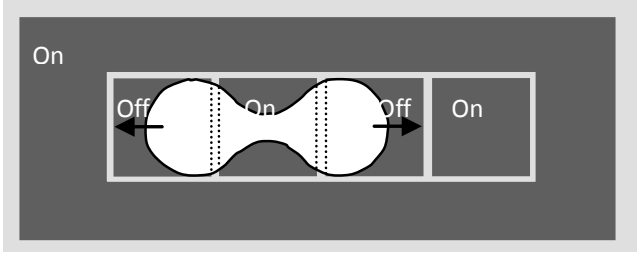

(b) Splitting

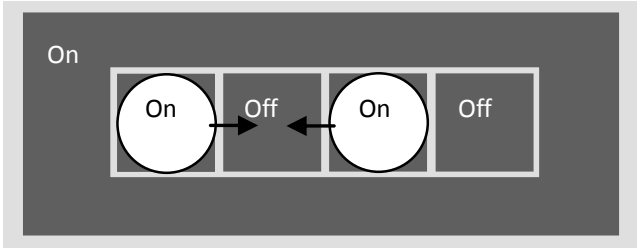

(c) Merging

Figure 2. Manipulation of bubbles in silicone oil by DEP utilizing the design of a surrounding electrode to confine the position of the bubbles. When applying voltage on selected electrodes among the confined four, oil will be driven onto the energized electrodes (shown "On") and push the bubble away from those electrodes to the non-energized electrodes (shown "Off").

In our experiment, the bubbles were squeezed between parallel plates as shown in Figure 1. The electrodes shown in Figure 2 were patterned on the bottom plate by wet etching of ITO on glass plates. The etched electrodes were then covered with a Teflon layer $(55 \mathrm{~nm})$ to reduce the surface energy for better bubble manipulations. The top plate was a blank ITO glass coated with 55-nm-thick Teflon. During device assembling, $20 \mathrm{cSt}$ silicone oil was first dispensed on the bottom plate. An air bubble with proper volume was then carefully dispensed with a pipette into the oil medium. Alternatively, the bubble could be created by DEP after the device was assembled. Finally, the top plate was placed onto the bottom plate with a 75 $\mu \mathrm{m}$-thick spacer. The square electrodes (Figure 2 ) on the bottom plate were $2 \mathrm{~mm} \times 2 \mathrm{~mm}$. The gap between electrodes was $40 \mu \mathrm{m}$.

\section{Bubble Transportation}

As shown in Figure 3, the bubble was successfully transported using DEP. A $0.3 \mu \mathrm{l}$ bubble was created in a $20 \mathrm{cSt}$ silicone oil medium in a $75 \mu \mathrm{m}$-high gap between parallel plates using DEP. The bubble was first positioned on the far left square of the electrode (electrode 1 in Figure $3(a)$ ). At this time, the surrounding electrode and electrodes 2 through 5 were switched on, while electrode 1 was switched off. Figure 3(b) demonstrates the movement of the bubble caused by switching on electrode 1 and switching off electrode 2, while other electrodes remained in the same state as before (Figure $3(\mathrm{a})$ ). By switching on electrode 1, the silicone oil was attracted by DEP. The bubble originally positioned on electrode 1 was then pushed away. Since electrode 2 was switched off, the bubble could move onto it. As illustrated in Figure 3(c), the bubble reached electrode 2 and stayed there. Electrode 2 was then switched on and electrode 3 was switched off, causing the bubble to move one electrode further to the right as shown in Figure 3(d) and (e). Similarly, by repeatedly switching on and off appropriate electrodes, the bubble was transported until finally reaching the far right electrode 5 .
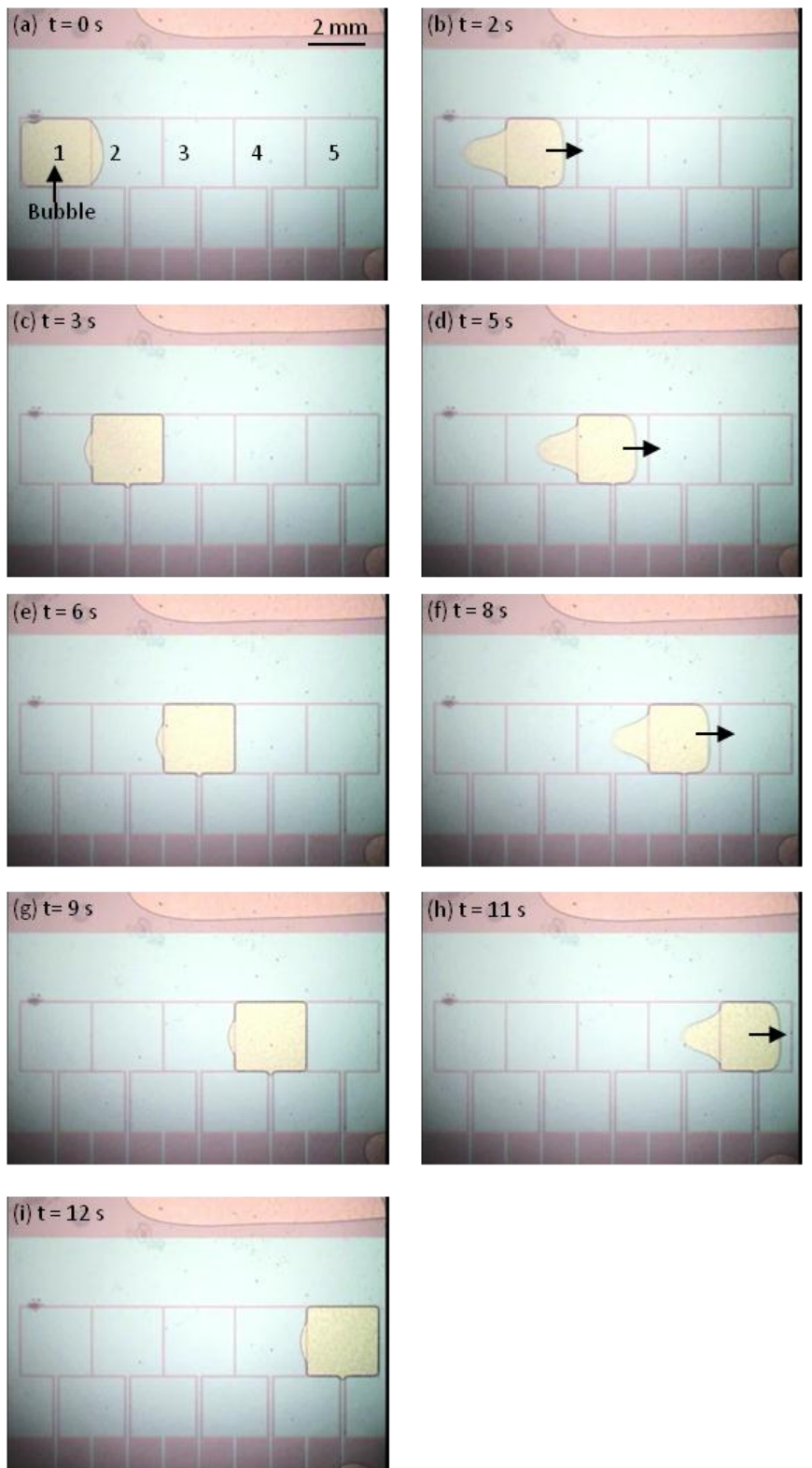

Figure 3. A $0.3 \mu \mathrm{l}$ bubble transported in $20 \mathrm{cSt}$ silicone oil between parallel plates spaced $75 \mu \mathrm{m}$. 
In this experiment, we used $570 \mathrm{~V}_{\mathrm{DC}}$ to drive the $0.3 \mu \mathrm{l}$ silicone oil droplet on $2 \mathrm{~mm}$-wide electrodes between a $75 \mu \mathrm{m}$-high gap. In theory (from Equation (1)), $A C$ voltages have been shown to be able to be used in bubble actuations. The DEP force is proportional to the width of the electrode and inversely propositional to the gap height. As such, pumping a bubble in a higher gap requires a higher voltage. Therefore, by changing the device dimensions, the required voltage could be estimated from Equation (1). Moreover, as discussed in the study of dielectric droplet manipulation by DEP, different driving voltages move the bubbles at different velocities. For the case shown in Figure 3, $570 V_{D C}$ pumped the bubble at a velocity $\sim 0.5 \mathrm{~mm} / \mathrm{s}$. A larger voltage could generate faster bubble transportation. However, the applied voltage should be lower than what could break down the $20 \mathrm{cSt}$ silicone oil $(13.8 \mathrm{~V} / \mathrm{mm}$ dielectric strength from Dow Corning $200^{\circ}$ Fluid data sheet). Conversely, by decreasing the applied voltage, the bubble could be moved at a slower velocity. Here, the bubble transportation was observed at $300 \mathrm{~V}_{\mathrm{DC}}$. The velocity and voltage relationship for driving dielectric droplets including silicone oil has been previously reported [16].
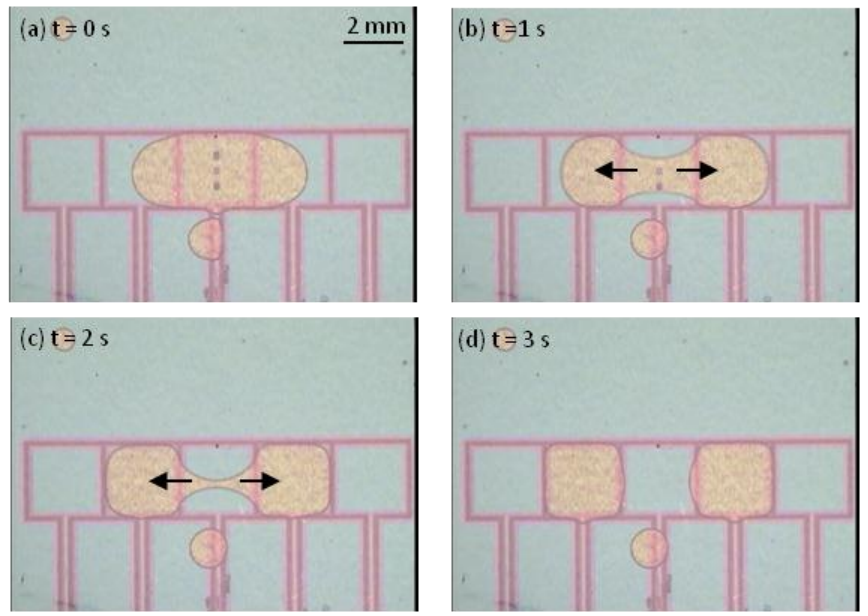

Figure 4. A $0.6 \mu \mathrm{l}$ bubble split into two bubbles in $20 \mathrm{cSt}$ silicone oil between parallel plates spaced $75 \mu \mathrm{m}$.

\section{Bubble Splitting}

In addition to bubble transportation, we also investigated bubble splitting. To achieve a successful split, a larger bubble was created. As shown in Figure 4(a), a $0.6 \mu$ bubble in a $20 \mathrm{cSt}$ silicone oil medium in a 75 - $\mu \mathrm{m}$-thick gap between parallel plates was prepared and positioned on electrodes 2-4, which were switched off, while electrodes 1, 5 and the surrounding electrode were switched on. Then, we split the bubble by switching on electrode 3. The DEP force occurring on electrode 3 attracted oil to it and pushed the bubble to the two adjacent off electrodes (electrode 2 and 4). It caused bubble necking (Figure 4(b) and (c)) and finally bubble splitting (Figure 4 (d)). The volume of the two split bubbles were similar and around $0.3 \mu$ l.

The voltage applied in Figure 4 to split the bubble was $570 \mathrm{~V}_{\mathrm{DC}}$. As discussed above, the value of the applied voltage affects the velocity of the bubble motion. Therefore, voltage also determines the splitting time. Our preliminary study showed that splitting could be achieved at the same voltage as bubble transportation. A systematic study will be performed to determine the required conditions for bubble splitting.


Figure 5. Bubbles merging in $20 \mathrm{cSt}$ silicone oil between parallel plates spaced $75 \mu \mathrm{m}$.

\section{Bubble Merging}

Bubble merging is a relatively simple task among the three functions. Merging can be achieved simply by applying a sufficient voltage on the electrode between the two bubbles. As shown in Figure 5, the two previously split bubbles (Figure 4 (d) and Figure 5(a)) were first positioned on electrodes 2 and 4 , which were off while the other electrodes were on. To merge them, electrode 3 was switched off and electrodes 2 and 4 were switched on. The DEP force attracted oil onto electrodes 2 and 4 and pushed the bubbles toward the non-energized electrode 3 , resulting in the bubbles being merged by DEP. Figure 5(b) shows the initial movement of the bubbles, while the completed merge is shown in Figure $5(\mathrm{c}) .490 \mathrm{~V}_{\mathrm{DC}}$ was used in this experiment to merge two $0.3 \mu \mathrm{l}$ bubbles in $20 \mathrm{cSt}$ silicone oil in a $75 \mu \mathrm{m}$-high gap between parallel plates.

\section{Conclusion}

Transporting, splitting, and merging bubbles immersed in 20 cSt silicone oil along a $75 \mu \mathrm{m}$-high gap between two parallel plates were successfully 
demonstrated through the use of DEP. With the ability to manipulate bubbles in an inert and low volatile medium, the composition of the bubble would remain stable and not altered by the vapor from the medium. The driving voltages ( $570 \mathrm{~V}_{D C}$ and $490 \mathrm{~V}_{D C}$ ) in the experiments were not optimum values. Depending on the device dimension and bubble velocities, the required voltage could be reduced. Threshold voltages and the voltage-velocity relation will also be studied in the future. The proposed bubble actuation mechanism can be applied to digital gaseous lab-on-a-chip. This technique, which enables splitting one bubble into two or more bubbles in a low vapor oil environment, can help in the analysis of different kinds of materials, such as ion, aluminum and so on. For example, if we want to analyze the influence of oxygen on different types of ions, we could put an oxygen gas bubble in an oil environment, and then split this bubble into more bubbles for analysis of the influence of oxygen. Moreover, this technique provides a more useful platform than other platforms for transporting, splitting, and merging bubbles.

\section{Acknowledgement}

This work is partially supported by National Science Council, Taiwan, R.O.C. under grants NSC 98-2221-E-009- 266-MY3 and NSC 100-2120-M-009-009-.

\section{References}

[1] M. G. Pollack, R. B. Fair, and A. D. Shenderov, "Electrowetting-based actuation of liquid droplets for microfluidic applications," Applied Physics Letters, vol. 77, no. 11, pp. 1725-1726, 2000. doi: $10.1063 / 1.1308534$

[2] J. Lee, H. Moon, J. Fowler, T. Schoellhammer, and C. J. Kim, "Electrowetting and electrowetting-on-dielectric for microscale liquid handling," Sensors and Actuators A: Physical, vol. 95, no. 2-3, pp. 259-268, 2002. doi: $10.1016 /$ S0924-4247(01)00734-8

[3] R. B. Fair, "Digital microfluidics: Is a true Lab-on-a-chip possible?," Microfluidics and Nanofluidics, vol. 3, no. 3, pp. 245-281, 2007. doi: 10.1007/s10404-007-0161-8

[4] M. Abdelgawad and A. R. Wheeler, "The digital revolution: A new paradigm for microfluidics," Advanced Materials, vol. 21, no. 8, pp. 920-925, 2009. doi: $10.1002 / a d m a .200802244$

[5] L. Malic, D. Brassard, T. Veres, and M. Tabrizian, "Integration and detection of biochemical assays in digital microfluidic LOC devices," Lab on a Chip, vol. 10, no. 4, pp. 418-431, 2010. doi: $10.1039 /$ B917668C
[6] R. Sista, Z. Hua, P. Thwar, A. Sudarsan, V. Srinivasan, A. Eckhardt, M. Pollack, and V. Pamula, "Development of a digital microfluidic platform for point of care testing," Lab on a Chip, vol. 8, no. 12, pp. 2091-2104, 2008. doi: 10.1039/B814922D

[7] S. K. Fan, P. W. Huang, T. T. Wang, and Y. H. Peng, "Cross-scale electric manipulations of cells and droplets by frequency-modulated dielectrophoresis and electrowetting," Lab on a Chip, vol. 8, no. 8, pp. 1325-1331, 2008. doi: 10.1039/B803204A

[8] S. K. Fan, C. P. Chiu, and J. W. Lin, "Electrowetting on polymer dispersed liquid crystal," Applied Physics Letters, vol. 94, no. 16, p. 164109, 2009. doi: $10.1063 / 1.3125253$

[9] B. Berge and J. Peseux, "Variable focal lens controlled by an external voltage: An application of electrowetting," The European Physical Journal E, vol. 3, no. 2, pp. 159-163, 2000. doi: $10.1007 / \mathrm{s} 101890070029$

[10] S. K. Fan, C. P. Chiu, and P. W. Huang, "Transmittance tuning by particle chain polarization in electrowetting-driven droplets," Biomicrofluidics, vol. 4, no. 4, pp. 043011, 2010. doi: $10.1063 / 1.3516656$

[11] D. Y. Kim and A. J. Steckl, "Liquid-state field-effect transistors using electrowetting," Applied Physics Letters, vol. 90, no. 4, p. 043507, 2007. doi: $\underline{10.1063 / 1.2435508}$

[12] Y. Zhao and S. K. Cho, "Micro air bubble manipulation by electrowetting on dielectric (EWOD): Transporting, splitting, merging and eliminating of bubbles," Lab on a Chip, vol. 7, no. 2, pp. 273-280, 2007. doi: $10.1039 / \mathrm{B} 616845 \mathrm{~K}$

[13] M. C. Wu and P. Y. Chiou, "Optofluidics and optoelectronic tweezers," in 13th Microoptics Conferenece, Takamatsu, Japan, 2007, pp. 104-107.

[14]S. M. Yang, T. M. Yu, H. P. Huang, M. Y. Ku, S. Y. Tseng, C. L. Tsai, H. P. Chen, L. Hsu, and C. H. Liu, "Light-driven manipulation of picobubbles on a titanium oxide phthalocyanine-based optoelectronic chip," Applied Physics Letters, vol. 98, no. 15, pp. 153512, 2011. doi: $10.1063 / 1.3580760$

[15] S. K. Fan, Y. W. Hsu, and C. H. Chen, "Encapsulated droplets with metered and removable oil shells by electrowetting and dielectrophoresis," Lab on a Chip, vol. 11, no. 15, pp. 2500-2508, 2011. doi: 10.1039/C1LC20142E

[16] S. K. Fan, T. H. Hsieh, and D. Y. Lin, "General digital microfluidic platform manipulating dielectric and conductive droplets by dielectrophoresis and electrowetting," Lab on a Chip, vol. 9, no. 9, pp. 1236-1242, 2009. doi: $10.1039 / B 816535 A$ 
[17] R. G. Fax, M. Hurwitz, and J. R. Melcher, "Dielectrophoretic liquid expulsion," Journal of Spacecraft and Rockets, vol. 6, no. 9, pp. 961-967, 1969.

doi: $10.2514 / 3.29740$

[18] D. S. Guttman, M. Hurwitz, and J. R. Melcher, "Dielectrophoretic orientation," Journal of Spacecraft and Rockets, vol. 6, no. 1, pp. 25-32, 1969. doi: $\underline{10.2514 / 3.29526}$
[19] M. Hurwitz and J. R. Melcher, "Gradient stabilization of electrohydrodynamically oriented liquids," Journal of Spacecraft and Rockets, vol. 4, no. 7, pp. 864-871, 1967.

doi: $10.2514 / 3.28980$

[20] H. Pellat, "Mesure de la force agissant sur les diélectriques liquides non électrisés, placés dans un champ électrique," Comptes Rendus de l'Académie des Sciences, vol. 119, pp. 691-694, 1895. 